\title{
Prognostic Value of the Alcoholic Hepatitis Histologic Score in Korean Patients with Biopsy-Proven Alcoholic Hepatitis
}

\author{
Dong Hyeon Lee ${ }^{1}$, Youn I Choi ${ }^{1 *}$, Jeong Mo Bae ${ }^{2}$, Mee Soo Chang ${ }^{3}$, Sae Kyung Joo ${ }^{1}$, Yong Jin Jung ${ }^{1}$, Kook Lae Lee ${ }^{1}$, Byeong \\ Gwan $\mathrm{Kim}^{1}$, and Won Kim ${ }^{1}$ \\ ${ }^{I}$ Division of Gastroenterology and Hepatology, Department of Internal Medicine, Seoul Metropolitan Government Seoul National University \\ Boramae Medical Center, Seoul National University College of Medicine, ${ }^{2}$ Department of Pathology, Seoul National University Hospital, Seoul \\ National University College of Medicine, and ${ }^{3}$ Department of Pathology, Seoul Metropolitan Government Seoul National University Boramae \\ Medical Center, Seoul National University College of Medicine, Seoul, Korea
}

Background/Aims: The alcoholic hepatitis histologic score (AHHS) is a recently developed clinical model for predicting short-term mortality in Caucasian patients with alcoholic hepatitis (AH). The AHHS has not been extensively validated in other ethnic populations. This study validated the AHHS in a Korean patient cohort. Methods: We conducted a prospective cohort study of hospitalized Korean patients with AH between January 2010 and August 2017. Histopathological findings were assessed to determine the AHHS in all study subjects. Histopathological risk factors were examined by Cox regression analysis to predict overall survival (OS). Kaplan-Meier curves were plotted to assess the diagnostic performance of the AHHS. Results: We recruited a total of 107 patients with biopsy-proven $\mathrm{AH}$. None of the individual AHHS components were associated with 3-month mortality. However, the bilirubinostasis type and fibrosis severity were significantly associated with AH mortality beyond 6 months (all $p<0.05$, except fibrosis severity for 6 -month mortality) and OS (all $p<0.05$ ). The modified AHHS classification as a binary variable ( $<5$ vs $\geq 5$ ) was also associated with OS (hazard ratio, 2.88; 95\% confidence interval [Cl], 1.50 to 5.56; $\mathrm{p}=0.002$ ), and had higher predictive performance for OS (concordance index [C-index], 0.634; 95\% Cl, 0.561 to 0.707) than the original AHHS classification (mild vs moderate vs severe: C-index, 0.577; 95\% Cl, 0.498 to 0.656). This difference was statistically significant $(p=0.045)$. Conclusions: In this prospective Korean AH cohort, the modified AHHS was significantly associated with OS. Therefore, the AHHS might be a useful histological prognosticator for long-term prognosis in patients with nonsevere AH. (Gut Liver 2020;14:636643)

Key Words: Hepatitis, alcoholic; Alcohol-related disorder; Classification; Biopsy; Prognosis

\section{INTRODUCTION}

In developed countries, alcohol-related liver disease is a major cause of advanced liver disease and is the leading cause of death among those who abuse alcohol. ${ }^{1,2}$ The most severe form of alcohol-related liver disease is alcoholic hepatitis (AH), which is clinically characterized by jaundice and liver failure. ${ }^{3}$ As patients with $\mathrm{AH}$ exhibit higher rates of short-term mortality, there is a need for more accurate patient characterization and more targeted therapies. ${ }^{4}$ Although $\mathrm{AH}$ is often suspected based on clinical and biochemical findings, histological confirmation remains the only method of confirming the diagnosis. ${ }^{5}$ As such, reliable noninvasive tests for $\mathrm{AH}$ are clearly needed.

In order to predict clinical outcome in patients with $\mathrm{AH}$, a number of models have been proposed. The Maddrey discriminant function and the model for end-stage liver disease (MELD) score have been used to identify those patients most likely to benefit from pharmacological therapy., ${ }^{6,7}$ Other validated models include the ABIC (age, serum bilirubin, international normalized ratio, and serum creatine) score, the Lille score, and the Glasgow alcoholic hepatitis score, and the MAGIC (model for AH to

\footnotetext{
Correspondence to: Won Kim ${ }^{\mathrm{a}}$ and Byeong Gwan Kim ${ }^{\mathrm{b}}$

Division of Gastroenterology and Hepatology, Department of Internal Medicine, Seoul Metropolitan Government Seoul National University Boramae Medical Center, 20 Boramae-ro 5-gil, Dongjak-gu, Seoul 07061, Korea

${ }^{a}$ Tel: +82-2-870-2233, Fax: +82-2-831-2826, E-mail: drwon1@snu.ac.kr

${ }^{\mathrm{b}}$ Tel: +82-2-870-2217, Fax: +82-2-831-2826, E-mail: caskim@brmh.org

Received on June 16, 2019. Revised on September 29, 2019. Accepted on October 12, 2019. Published online May 29, 2020.

pISSN 1976-2283 eISSN 2005-1212 https://doi.org/10.5009/gnl19203

Dong Hyeon Lee and Youn I Choi contributed equally to this work as first authors.

*Current affiliation of Youn I Choi is Department of Gastroenterology, Gachon University Gil Medical Center, Incheon, Korea.

@ This is an Open Access article distributed under the terms of the Creative Commons Attribution Non-Commercial License (http://creativecommons.org/licenses/by-nc/4.0) which permits unrestricted non-commercial use, distribution, and reproduction in any medium, provided the original work is properly cited.
} 
grade severity in an Asian patient cohort) score. ${ }^{8-11}$ Although biochemical data can be used to predict clinical outcome, $\mathrm{AH}$ is likely the only chronic liver disease that lacks a validated histological classification.

Previous studies have shown that several individual histological parameters are associated with clinical outcome in patients with $\mathrm{AH} .{ }^{12-16}$ However, there was no attempt to develop a novel histological classification by integrating these parameters. Altamirano et al. ${ }^{17}$ recently proposed a new histological scoring system, named the alcoholic hepatitis histologic score (AHHS) for predicting short-term survival in patients with AH. The AHHS is based on several histological features, including degree of fibrosis, degree of neutrophil infiltration, type of bilirubinostasis, and presence or absence of megamitochondria. The AHHS is associated with disease severity and predicts the risk of death within 90 days after histological confirmation in patients with $\mathrm{AH}^{17}$

The aims of this study were to (1) assess the frequency of each AHHS histological feature among Korean patients with $\mathrm{AH}$ and (2) evaluate the prognostic value of the AHHS for predicting long-term survival as well as short-term survival in Korean patients with $\mathrm{AH}$.

\section{MATERIALS AND METHODS}

\section{Study cohort}

The longitudinal cohort for this study was derived from an ongoing, single-center AH registry. Subjects with clinical and biochemical evidence of AH between January 2010 and August 2017 were prospectively enrolled based on inclusion criteria. The criteria for eligibility for liver biopsy were as follows: (1) $\geq 18$ years old; (2) alcohol consumption $>60 \mathrm{~g} /$ day for men and $>40 \mathrm{~g} /$ day for women before admission; (3) recent onset of jaundice; (4) elevated levels of gamma-glutamyl transpeptidase; (5) elevated levels of transaminases with an aspartate aminotransferase/alanine aminotransferase ratio $>1$; and (6) an absence of other etiologies for chronic liver disease such as hepatitis B or C, autoimmune hepatitis, drug-induced liver injury, hemochromatosis, primary biliary cholangitis, or Wilson disease. The prospective study was conducted with those patients with histological confirmation of $\mathrm{AH}$ as evidenced by hepatocellular ballooning, steatosis, and neutrophil infiltration. Forty patients were not enrolled due to the presence of other causes of chronic liver disease (hepatitis $\mathrm{C}$ infection, $\mathrm{n}=16$; concomitant hepatitis $\mathrm{C} /$ human immunodeficiency virus [HIV] coinfection, $\mathrm{n}=3$; miliary tuberculosis, $n=1$; syphilitic hepatitis, $n=1$; hemochromatosis, $n=1$; or drug-induced liver injury, $n=4$ ) or failure to fulfill histologic criteria for $\mathrm{AH}(\mathrm{n}=14)$. Based upon inclusion and exclusion criteria along with histological confirmation of $\mathrm{AH}$, we enrolled a total of 107 patients in this study.

During the study, all patients received general supportive care including nutrient supplementation, enteral or parenteral nutrition if needed, and administration of B complex vitamins. Those patients that developed complications during hospitalization were treated according to current international practice guidelines. ${ }^{18-21}$ Patients with an Maddrey discriminant function score $\geq 32$ were treated with oral prednisolone ( $40 \mathrm{mg}$ daily) for 4 weeks, followed by a 2 -week taper period. If oral dosing was not tolerated, methylprednisolone (intravenous) with equivalent efficacy (32 mg each 24 hours) was administered. The treatment response to corticosteroids was assessed at day 7 using the Lille score with a cutoff of $0.45 .{ }^{11}$ Patients with contraindications to corticosteroids were treated with pentoxifylline. We obtained written informed consent from each patient in the study cohort. This study was conducted according to the provisions in the Declaration of Helsinki for the participation of human subjects in research and was approved by the Institutional Review Board of Boramae Medical Center (IRB number: 16-2013-45). The trial was registered at clinicaltrials.gov (NCT 01943318).

\section{Histological and clinical assessment}

Electronic medical records were used to obtain demographic, clinical, and laboratory parameters. Liver samples were obtained between days 1 and 7 after admission, either percutaneously or via a transjugular approach depending on the presence of variceal bleeding, ascites, or coagulopathy. Samples were fixed in 4\% formalin, then embedded in paraffin. All liver biopsies were assessed and reviewed by a single, experienced liver pathologist (J.M.B.). Detailed histological analyses were performed both prospectively and retrospectively and included the following observations: (1) presence or absence of hepatocellular damage or ballooning; (2) degree of polymorphonuclear leukocyte infiltration (absent, mild, moderate, or severe); (3) presence or absence of Mallory bodies; (4) degree of steatosis (absent, mild, moderate, or severe); (5) presence and location of bilirubinostasis (absent, hepatocellular bilirubinostasis, canalicular or ductular bilirubinostasis, or mixed hepatocellular plus canalicular or ductular bilirubinostasis); (6) presence or absence of megamitochondria; and (7) stage of fibrosis (no fibrosis, portal fibrosis, expansive fibrosis, bridging fibrosis, or cirrhosis). ${ }^{12-17}$ Diagnosis of AH was based on the overall pattern of histological injury including hepatocellular damage (presence of Mallory bodies and hepatocellular ballooning), inflammatory infiltrate polymorphonuclear leukocytes, and pericellular fibrosis. ${ }^{8-22}$ In order to evaluate a possible association between short-term outcomes and the AHHS score, we graded the severity of fibrosis, type of bilirubinostasis, degree of neutrophil infiltration, and presence of megamitochondria. ${ }^{17}$

\section{Endpoints and assessments}

The endpoints included overall survival (OS) and mortality at 3 months, 6 months, and 1 year from the date of biopsy. OS was measured from the date of biopsy until the date of liver transplantation (LT) or death from any cause. When study par- 
ticipants were lost to follow-up before LT or death, those data were censored with respect to the date of their last clinic visit. The data cutoff date was May 10, 2018. All death events were verified using mortality data from the Ministry of the Interior and Safety of South Korea. Short-term mortality was defined as death within 3 months, whereas long-term mortality was defined as death beyond 6 months.

\section{Statistical analysis}

Median and interquartile range (IQR) were used to describe continuous variables, while means of counts and percentages were used to describe categorical variables. Logistic regression analysis was performed to identify histological features associated with 3-month, 6-month, and 1-year mortality. Associations between histological features and OS, and between the AHHS and OS, were assessed using Cox proportional hazard models. Harrell's concordance-index (C-index) was calculated to validate the prognosis-predictive performance of the AHHS based on the histological classification system. The "compareC" package was used to determine significance between C-indices of the anatomic and prognostic staging models. ${ }^{23}$ Kaplan-Meier curves were used to generate survival data; statistical significance was determined using log-rank tests. p-values $<0.05$ were considered significant. All statistical analyses were performed using SPSS version 20.0 (IBM Corp., Armonk, NY, USA) and the R statistical programming environment, version 3.5.1 (R Foundation for Statistical Computing, Vienna, Austria).

\section{RESULTS}

\section{Characteristics of study participants}

This study included 107 patients with $\mathrm{AH}$ as confirmed by histological confirmation. Demographic, clinical, and biochemical data for the study population are presented in Table 1 . The median age was 52 years (IQR, 43 to 59 years); 85 patients (79.4\%) were male. The median time interval between admission and liver biopsy was 3 days (IQR, 1 to 4 days). One patient experienced acute anemia after percutaneous liver biopsy. However, he was discharged safely after conservative management without further deterioration.

A total of 31 patients (29.0\%) were treated with corticosteroids; among these, 28 patients $(90.3 \%)$ responded to steroid therapy in terms of the Lille score $(<0.45)$ and completed the scheduled treatment for 28 days. Four patients with contraindications to corticosteroids were treated with pentoxifylline as an alternative therapy. Median follow-up duration was 68 weeks (IQR, 22 to 130 weeks), and the observed overall 3-month, 6-month, and 1-year mortality rates were 12.1\%, 19.6\%, and $30.8 \%$, respectively. After discharge, only 20 patients (18.7\%) achieved alcohol abstinence until the last follow-up and 43 patients $(40.2 \%)$ were readmitted due to alcohol recidivism within 3 months.

\section{Histological factors associated with mortality}

First, we assessed the relationships between histological features and 3-month mortality (Supplementary Table 1). Univariate and multivariate analyses revealed that none of the AHHS components or any other histological features at admission were significantly associated with 3-month mortality. However, bilirubinostasis type and fibrosis severity were significantly associated with long-term mortality greater than 6 months (Supplementary Table 2). Ductular and/or canalicular plus hepatocellular bilirubinostasis was associated with 6-month mortality (odds ratio, 3.88; 95\% confidence interval [CI], 1.39 to 10.83 ; $\mathrm{p}=0.010$ ) and 1-year mortality (adjusted odds ratio, 7.05; 95\% CI, 2.34 to 21.23 ; $\mathrm{p}=0.001$ ), and bridging and/or cirrhosis was significantly associated with 1-year mortality (adjusted odds ratio, 25.96; 95\% CI, 3.13 to $215.00 ; \mathrm{p}=0.003$ ).

OS was significantly associated with the bilirubinostasis type (ductular and/or canalicular plus hepatocellular vs no or hepatocellular only: adjusted hazard ratio [HR], 3.37; 95\% CI, 1.79 to 6.34; $\mathrm{p}<0.001)$ and fibrosis severity (no/portal and/or expansive vs bridging and/or cirrhosis: adjusted HR, 5.28; 95\% CI, 2.04 to 13.70; $\mathrm{p}=0.001$ ) (Table 2).

\section{Prognostic assessment based on the AHHS}

Fifty patients (46.7\%) died and two patients (1.9\%) received LT during the follow-up period. Because more than half of patients were still alive without LT during the follow-up duration, we could not estimate the median OS. Among the 50 deaths, 19 occurred in the hospital and 16 (84.2\%) succumbed to liver-related events (seven for bleeding, five for hepatorenal syndrome, and four for infection). Patients classified into the mild disease group (AHHS 0-3 points) had better prognosis than those classified into the moderate (4-5 points) and severe (6-9 points) groups. These differences, however, were not statistically significant (moderate vs mild: HR, 1.57; 95\% CI, 0.67 to 3.66; $\mathrm{p}=0.300$; severe vs mild: $\mathrm{HR}, 2.37$; $95 \% \mathrm{CI}, 1.00$ to 5.59 ; $\mathrm{p}=0.050$; and severe vs moderate: $\mathrm{HR}, 1.51 ; 95 \% \mathrm{CI}, 0.83$ to 2.73 ; $\mathrm{p}=0.176)$ (Table 3). The C-index of the AHHS was $0.577(95 \%$ CI, 0.498 to 0.656 ) (Table 4).

Next, we used an alternative cutoff value ( $<5$ vs $\geq 5$ ) proposed by Altamirano et al. ${ }^{17}$ for sequential use of noninvasive scoring systems (i.e., MELD and ABIC scores) and the AHHS. Using this cutoff, the AHHS successfully defined two subgroups with different survival rates (HR, 3.16; 95\% CI, 1.65 to 6.07; $\mathrm{p}=0.001)$. The C-index of the modified AHHS classification system ( $<5$ vs $\geq 5$ : C-index, 0.634 ; 95\% CI, 0.561 to 0.707 ) was significantly better than that of the original AHHS classification system (mild vs moderate vs severe: C-index, 0.577; 95\% CI, 0.498 to $0.656 ; p=0.045)$. The log-rank test showed that the modified AHHS classification as a binary variable $(\mathrm{p}<0.001)$ provided better discrimination between subgroup differences than the original AHHS classification ( $\mathrm{p}=0.106)$ (Fig. 1). 
Table 1. Baseline Patient Characteristics $(n=107)$

\begin{tabular}{|c|c|}
\hline Characteristic & Value \\
\hline Age, yr & $52(43-59)$ \\
\hline Male sex & $85(79.4)$ \\
\hline Body weight, $\mathrm{kg}$ & $59.8(52.0-71.1)$ \\
\hline Alcohol intake, g/day & $80(45-113)$ \\
\hline Use of corticosteroids & $31(29.0)$ \\
\hline Length of biopsy specimen, $\mathrm{cm}$ & $1.4(1.2-1.6)$ \\
\hline \multicolumn{2}{|l|}{ Clinical decompensation at admission } \\
\hline Ascites & $46(43.0)$ \\
\hline Well-controlled & $35(32.7)$ \\
\hline Refractory & $11(10.3)$ \\
\hline Encephalopathy & $1(0.9)$ \\
\hline \multicolumn{2}{|l|}{ Infection } \\
\hline Spontaneous bacterial peritonitis & $6(5.6)$ \\
\hline Pneumonia & $2(1.9)$ \\
\hline UTI & $1(0.9)$ \\
\hline Colitis & $1(0.9)$ \\
\hline Sinusitis & $1(0.9)$ \\
\hline Cellulitis & $1(0.9)$ \\
\hline Miscellaneous & $9(8.4)$ \\
\hline \multicolumn{2}{|c|}{ Laboratory and hemodynamic parameters } \\
\hline Hemoglobin, g/dL & $11.3(9.6-12.9)$ \\
\hline Leukocyte count, $\times 10^{9} / \mathrm{L}$ & $6.1(4.6-9.1)$ \\
\hline Platelet count, $\times 10^{9} / \mathrm{L}$ & $132(81-190)$ \\
\hline Aspartate aminotransferase, $\mathrm{U} / \mathrm{L}$ & $86(58-142)$ \\
\hline Alanine aminotransferase, $\mathrm{U} / \mathrm{L}$ & $32(22-57)$ \\
\hline Serum sodium, mmol/L & $136(132-139)$ \\
\hline Serum albumin, $g / d L$ & $3.3(2.8-3.9)$ \\
\hline Serum creatinine, $\mathrm{mg} / \mathrm{dL}$ & $0.7(0.6-0.9)$ \\
\hline Serum bilirubin, mg/dL & $4.1(1.0-8.5)$ \\
\hline International normalized ratio & $1.32(1.07-1.61)$ \\
\hline Liver volume, $\mathrm{cm}^{3 *}$ & $1,842(1,355-2,440)$ \\
\hline \multicolumn{2}{|l|}{ AH severity scores at admission } \\
\hline MDF score & $12.8(-1.3-37.3)$ \\
\hline MELD score & $14(8-22)$ \\
\hline ABIC score & $7.0(6.4-7.6)$ \\
\hline \multicolumn{2}{|l|}{ ABIC class } \\
\hline $\mathrm{A}(<6.71)$ & $38(35.5)$ \\
\hline В (6.71-8.99) & $63(58.9)$ \\
\hline$C(\geq 9)$ & $6(5.6)$ \\
\hline \multicolumn{2}{|l|}{ Histological features at admission } \\
\hline \multicolumn{2}{|l|}{ Fibrosis stage } \\
\hline No fibrosis or portal fibrosis & $17(15.9)$ \\
\hline Expansive fibrosis & $14(13.1)$ \\
\hline Bridging fibrosis or cirrhosis & $76(71.0)$ \\
\hline
\end{tabular}

Table 1. Continued

\begin{tabular}{lc}
\hline \multicolumn{1}{c}{ Characteristic } & Value \\
\hline Steatosis, \% & $37(34.6)$ \\
$5-33$ & $26(24.3)$ \\
$33-66$ & $44(41.1)$ \\
$>66$ & \\
Mallory bodies & $44(41.1)$ \\
None or occasional & $63(58.9)$ \\
Marked & \\
Bilirubinostasis & $45(42.1)$ \\
None & $31(29.0)$ \\
Hepatocellular & $4(3.7)$ \\
Canalicular and/or ductular & $27(25.2)$ \\
Hepatocellular plus canalicular and/or ductular & \\
Ballooning & $71(66.4)$ \\
Occasional & $36(33.6)$ \\
Marked & \\
PMN infiltration & $93(86.9)$ \\
None/mild & $14(13.1)$ \\
Severe & \\
Megamitochondria & $20(18.7)$ \\
No & \\
Yes & \\
\hline
\end{tabular}

Data are presented as the median (interquartile range) or number (\%). UTI, urinary tract infection; AH, alcoholic hepatitis; MDF, Maddrey discriminant function; MELD, model for end-stage liver disease; ABIC, age, serum bilirubin, international normalized ratio, and serum creatinine; PMN, polymorphonuclear neutrophil.

*Baseline total liver volume was measured using Aquarius iNtuition software (TeraRecon, Houston, TX, USA) in 52 patients.

\section{Sequential use of clinical scoring systems and the AHHS}

The modified AHHS ( $<5$ vs $\geq 5$ ) was independently associated with OS after adjusting for ABIC $(\mathrm{p}<0.001)$ or MELD $(\mathrm{p}<0.001)$ score (Supplementary Table 3). Therefore, we investigated whether the sequential use of clinical and histological scores improved the prognostic performance for predicting survival in patients with AH. Since there were only six patients in the ABIC class C, the analysis for patients with ABIC class $C$ was not conducted. The sequential use of the AHHS $(<5$ vs $\geq 5)$ did not improve the prognostic assessment in patients with a MELD score $\geq 21(\mathrm{p}=0.262)$. However, the AHHS ( $<5 \mathrm{vs} \geq 5$ ) did successfully discriminate two subgroups according to the risk of death in patients with ABIC class A and B (ABIC score $<9)(p=0.001)$ (Fig. 2A), and patients with MELD score $<21$ ( $\mathrm{p}=0.006$ ) (Fig. 2B).

\section{DISCUSSION}

In this prospective Korean AH cohort, we validated the prognosis-predictive value of the recently proposed AHHS by assessing OS in histologically confirmed patients. Contrary to the 
Table 2. Univariate and Multivariate Analyses of Histological Features Associated with Overall Survival in Patients with Alcoholic Hepatitis

\begin{tabular}{|c|c|c|c|c|c|c|c|c|c|}
\hline \multirow{2}{*}{ Histologic features } & \multicolumn{3}{|c|}{$\begin{array}{l}\text { Univariate Cox regression } \\
\text { (events, } n=52 \text { ) }\end{array}$} & \multicolumn{3}{|c|}{$\begin{array}{l}\text { Multivariate Cox regression } \\
\text { (events, } n=52 \text { ) }\end{array}$} & \multicolumn{3}{|c|}{$\begin{array}{l}\text { Multivariate Cox regression } \\
\text { (events, } n=52 \text { ) }\end{array}$} \\
\hline & $\mathrm{HR}$ & $95 \% \mathrm{CI}$ & $\mathrm{p}$-value & $\mathrm{HR}$ & $95 \% \mathrm{CI}$ & p-value & HR & $95 \% \mathrm{CI}$ & $\mathrm{p}$-value \\
\hline \multicolumn{10}{|l|}{ Fibrosis } \\
\hline No/portal and/or expansive & 1.00 & Reference & & 1.00 & Reference & & 1.00 & Reference & \\
\hline Bridging and/or cirrhosis & 5.66 & $2.24-14.31$ & $<0.001$ & 5.28 & $2.04-13.70$ & 0.001 & 5.69 & $2.22-14.57$ & $<0.001$ \\
\hline \multicolumn{10}{|l|}{ Bilirubinostasis } \\
\hline No or hepatocellular only & 1.00 & Reference & & 1.00 & Reference & & 1.00 & Reference & \\
\hline Ductular and/or canalicular & 4.20 & $1.24-14.14$ & 0.021 & 3.44 & $0.99-12.02$ & 0.052 & 5.31 & $1.49-18.88$ & 0.010 \\
\hline $\begin{array}{l}\text { Ductular and/or canalicular plus } \\
\text { hepatocellular }\end{array}$ & 4.02 & $2.27-7.13$ & $<0.001$ & 3.37 & $1.79-6.34$ & $<0.001$ & 4.10 & $2.23-7.55$ & $<0.001$ \\
\hline Mild & 1.00 & Reference & & & & & 1.00 & Reference & \\
\hline Moderate/severe & 1.40 & $0.70-2.80$ & 0.345 & & & & 0.63 & $0.29-1.36$ & 0.235 \\
\hline Megamitochondria & & & & - & - & - & & & \\
\hline No & 1.00 & Reference & & & & & 1.00 & Reference & \\
\hline Yes & 1.40 & $0.75-2.63$ & 0.295 & & & & 1.00 & $0.51-1.97$ & 0.997 \\
\hline Ballooning & & & & & & & - & - & - \\
\hline Occasional & 1.00 & Reference & & 1.00 & Reference & & & & \\
\hline Marked & 2.60 & $1.50-4.51$ & 0.001 & 0.98 & $0.52-1.85$ & 0.944 & & & \\
\hline Mallory bodies & & & & & & & - & - & - \\
\hline No & 1.00 & Reference & & 1.00 & Reference & & & & \\
\hline Yes & 2.57 & $1.37-4.80$ & 0.003 & 1.64 & $0.83-3.24$ & 0.153 & & & \\
\hline $5-33$ & 1.00 & Reference & & & & & & & \\
\hline $33-66$ & 1.63 & $0.80-3.32$ & 0.177 & & & & & & \\
\hline$>66$ & 1.02 & $0.52-1.99$ & 0.954 & & & & & & \\
\hline
\end{tabular}

HR, hazard ratio; CI, confidence interval; PMN, polymorphonuclear neutrophil.

Table 3. Cox Model Results for Overall Patient Survival Based on the AHHS

\begin{tabular}{lccc}
\hline \multirow{2}{*}{ Histologic features } & \multicolumn{3}{c}{ Cox regression (events, $\mathrm{n}=52)$} \\
\cline { 2 - 4 } & HR & $95 \% \mathrm{CI}$ & $\mathrm{p}$-value \\
\hline AHHS & & & \\
Mild (0-3) & 1.00 & Reference & \\
Moderate (4-5) & 1.57 & $0.67-3.66$ & 0.300 \\
Severe (6-9) & 2.37 & $1.00-5.59$ & 0.050 \\
Moderate (4-5) & 1.00 & Reference & \\
Severe (6-9) & 1.51 & $0.83-2.73$ & 0.176 \\
AHHS & & & \\
AHHS $<5$ & 1.00 & Reference & \\
AHHS $\geq 5$ & 3.16 & $1.65-6.07$ & 0.001 \\
\hline
\end{tabular}

$\overline{\mathrm{AHHS}}$, alcoholic hepatitis histologic score; HR, hazard ratio; CI, confidence interval.

previous studies, ${ }^{17,24}$ our analyses indicate that none of the integral components of the AHHS were associated with 3-month mortality. By contrast, the type of bilirubinostasis and severity
Table 4. Ranking of Classification by the C-Index

\begin{tabular}{lccc}
\hline \multicolumn{1}{c}{ Histological features } & C-index & $95 \%$ CI & p-value \\
\hline AHHS (mild vs moderate vs severe) & 0.577 & $0.498-0.656$ & 0.045 \\
AHHS (<5 vs $\geq 5$ ) & 0.634 & $0.561-0.707$ & \\
\hline
\end{tabular}

C-index, concordance index; CI, confidence interval; AHHS, alcoholic hepatitis histologic score.

of fibrosis were significantly associated with long-term survival greater than 6 months. The bilirubinostasis pattern and fibrosis stage were also significantly associated with OS. The AHHS was significantly associated with OS when a cutoff of 5 points was used. Therefore, these results suggest that long-term survival (as compared to short-term survival) may be predicted by the AHHS in patients with nonsevere $\mathrm{AH}$.

Previous studies reported that the presence of bilirubinostasis is an independent predictor of short-term mortality in AH. ${ }^{14,15,17,24}$ Although bilirubinostasis was not associated with 3-month mortality in our cohort, it was significantly associated with 6-month and 1-year mortality. Since the presence of bili- 

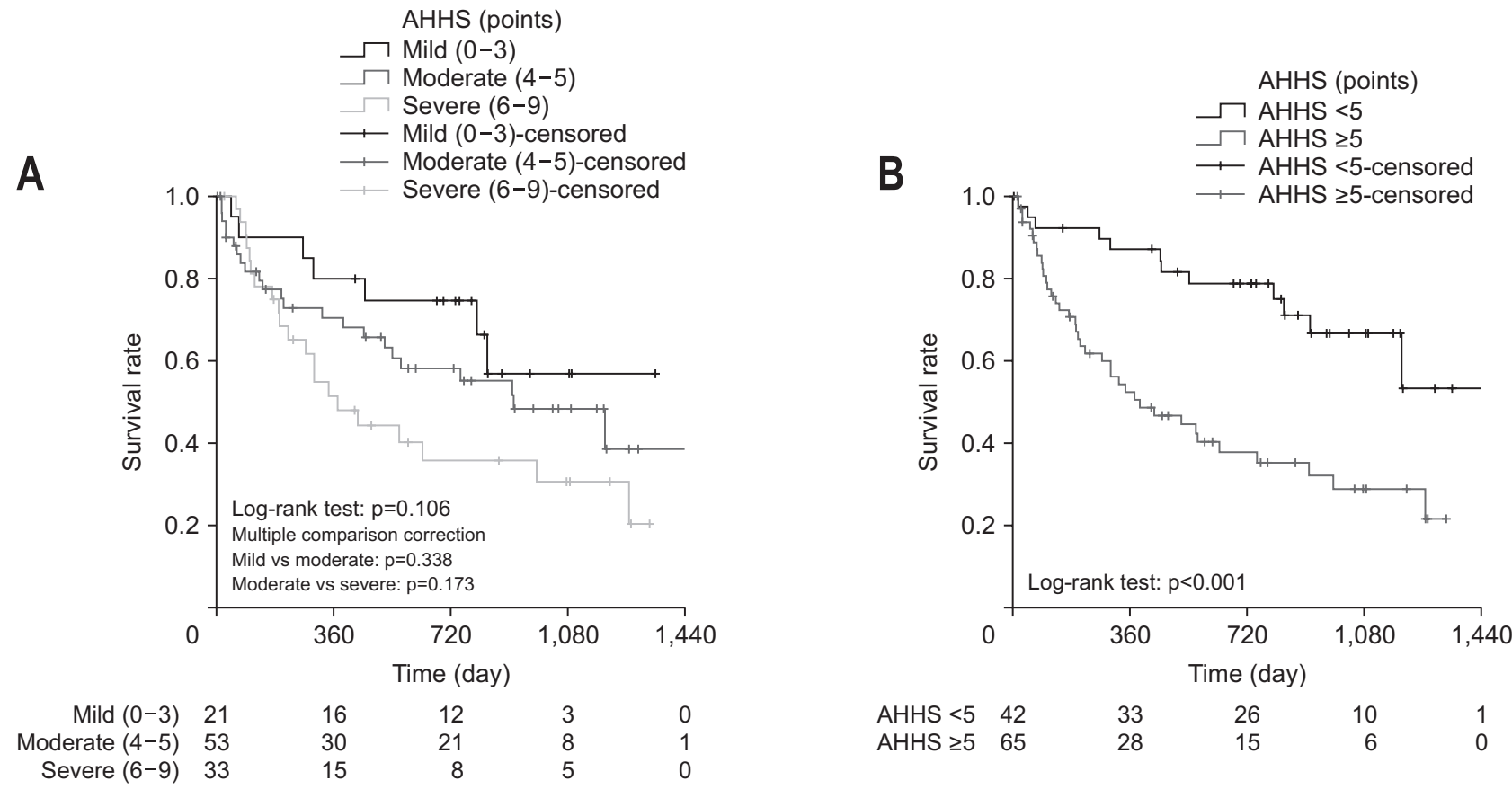

Fig. 1. Overall survival probability of patients with alcoholic hepatitis according to the original alcoholic hepatitis histologic score (AHHS) (A) and the modified AHHS with a cutoff of 5.0 (B).
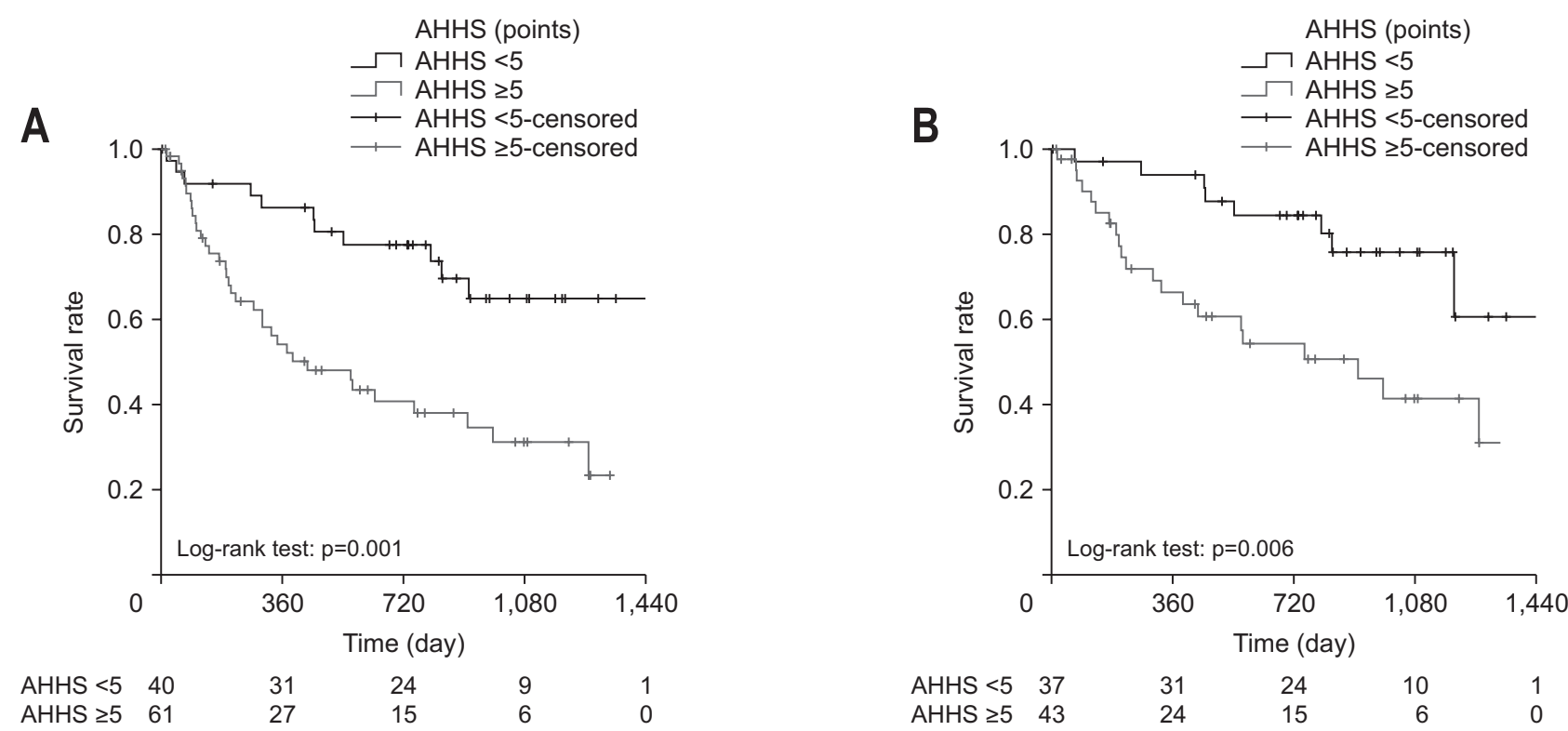

Fig. 2. Overall survival probability of patients with alcoholic hepatitis (AH) and ABIC class A and B (ABIC score $<9$ ) (A) and patients with AH and a MELD score $<21$ (B) according to the modified AHHS with a cutoff of 5.0.

ABIC, age, serum bilirubin, international normalized ratio, and serum creatinine; MELD, model for end-stage liver disease; AHHS, alcoholic hepatitis histologic score.

rubinostasis is a surrogate marker for impairment in hepatocellular bile transport and hepatic bile flow, it is common to find bilirubinostasis in patients with severe $\mathrm{AH}$. Several studies have reported that development of bacterial infection and sepsis was associated with bilirubinostasis type. ${ }^{15-17}$ Fibrosis severity was associated with 1-year survival in these patients. This can be attributed to the fact that advanced fibrosis can lead to cirrhosis- related complications and portal hypertension. ${ }^{25}$ Further studies are warranted to identify key molecular drivers and optimal therapeutic targets for bilirubinostasis and fibrosis in patients with $\mathrm{AH}$.

A novel histological scoring system was developed by $\mathrm{Al}-$ tamirano et al. ${ }^{17}$ to predict short-term mortality in patients with $\mathrm{AH}$. The 3-month mortality rate in the discovery cohort was 
successfully predicted by AHHS with an area under the receiver operating characteristic curve of 0.77 (95\% CI, 0.71 to 0.83). The present study reveals that the AHHS can be used to predict OS using a cutoff of 5.0 points with a C-index of 0.634 (95\% CI, 0.561 to 0.707 ), although the prognostic performance of the AHHS in our Korean cohort was inferior to that of the original discovery cohort. We also found that the AHHS was independently associated with OS even after adjusting for some clinical scoring systems, which is consistent with the previous study. ${ }^{17}$ We used the AHHS to improve the prognostic stratification of $\mathrm{AH}$ patients with $\mathrm{ABIC}$ class $\mathrm{A}$ and $\mathrm{B}$ (ABIC score <9) showing low to moderate risk of mortality. ${ }^{8}$ The AHHS also provided a more accurate prediction of survival for $\mathrm{AH}$ patients with a MELD score $<21$, the low risk of mortality group. ${ }^{26}$ Sequential use of the noninvasive clinical scoring systems and the AHHS could help to discriminate patients with relatively high risk of death from those with low to moderate risk of death.

Our study is the first to evaluate the ability of AHHS to evaluate long-term prognosis in patients with $\mathrm{AH}$. In addition, a major strength of this study is that it is the first external validation of AHHS in a Korean population with histologically confirmed $\mathrm{AH}$. The impact of race and ethnicity on $\mathrm{AH}$ is not clear. However, this independent external validation of the AHHS in the Korean population is indispensable because of known ethnic significant differences in mitochondrial aldehyde dehydrogenase activity and obesity prevalence. ${ }^{27,28}$ The present study also is the first to clarify the association of the AHHS with OS, not restricted to liver-related mortality. Although a previous study performed survival analyses in some subgroups, it did not perform survival analyses for all patients. ${ }^{17}$ Another strength of our study lies in the fact that it affirms the usefulness of the AHHS and sequential use of noninvasive clinical scoring systems for risk stratification, demonstrating that using a cutoff of 5.0 points derived for sequential analyses was more effective and simpler for risk stratification than the original AHHS classification (mild vs moderate vs severe).

There are certain limitations that should be considered in the present study. First, the majority of patients had relatively wellpreserved hepatic reserve (median MELD score, 14; median ABIC score, 7.0), and the 3-month mortality rate was much lower in the current study than in the previous discovery and validation studies. ${ }^{17,24}$ The disease severity of the observed population was different in our study than in the previous studies; therefore, the AHHS might not accurately predict 3-month mortality in our study despite the scoring system that was designed to predict short-term mortality. Second, we did not consider potential differences in interobserver variability in the present and previous studies. Significant interobserver variability among individual pathologists was found in the previous study. ${ }^{29}$ Although the majority of patients in our study exhibited mild to moderate $\mathrm{AH}$, the proportion of patients with severe polymorphonuclear leukocyte infiltration, known as a favorable prognostic marker, was lower than in the previous study (13.1\% vs 33\%). These findings suggest that there could be significant interobserver variability among pathologists in the present and previous studies.

In summary, we observed that the AHHS accurately predicted OS and mortality beyond 6 months although the integral components of the AHHS were not significantly associated with 3-month mortality. Our results suggest that the AHHS is a useful tool to predict long-term prognosis rather than short-term mortality in Korean patients with relatively nonsevere $\mathrm{AH}$.

\section{CONFLICTS OF INTEREST}

No potential conflict of interest relevant to this article was reported.

\section{ACKNOWLEDGEMENTS}

This work was supported by Seoul Metropolitan Government Seoul National University (SMG-SNU) Boramae Medical Center (grant number: 03-2019-2).

\section{AUTHOR CONTRIBUTIONS}

Study concept and design: D.H.L., Y.I.C., W.K. Data acquisition: Y.I.C., J.M.B., S.K.J. Data analysis and interpretation: D.H.L., W.K. Drafting of the manuscript: D.H.L., Y.I.C. Critical revision of the manuscript for important intellectual content: M.S.C., Y.J.J., K.L.L. Statistical analysis: D.H.L., Y.I.C. Obtained funding: B.G.K. Study supervision: B.G.K., W.K. Final approval of the article: all authors.

\section{ORCID}

Dong Hyeon Lee

Youn I Choi

Jeong Mo Bae

Mee Soo Chang

Sae Kyung Joo

Yong Jin Jung

Kook Lae Lee

Byeong Gwan Kim

Won Kim https://orcid.org/0000-0003-2044-6854 https://orcid.org/0000-0001-6561-6752 https://orcid.org/0000-0003-0462-3072 https://orcid.org/0000-0002-0948-799X https://orcid.org/0000-0002-4615-7607 https://orcid.org/0000-0001-8785-2254 https://orcid.org/0000-0001-6676-9451 https://orcid.org/0000-0003-0965-226X https://orcid.org/0000-0002-2926-1007

\section{REFERENCES}

1. Mathurin P, Bataller R. Trends in the management and burden of alcoholic liver disease. J Hepatol 2015;62(1 Suppl):S38-S46.

2. Lee JY, Cho Y, Hong MH, et al. Incidence, inhospital mortality, and readmission among patients with alcoholic hepatitis in Korea a nationwide study. J Gastroenterol Hepatol 2019;34:747-754.

3. Lucey MR, Mathurin P, Morgan TR. Alcoholic hepatitis. N Engl J 
Med 2009;360:2758-2769.

4. Crabb DW, Bataller R, Chalasani NP, et al. Standard definitions and common data elements for clinical trials in patients with alcoholic hepatitis: recommendation from the NIAAA Alcoholic Hepatitis Consortia. Gastroenterology 2016;150:785-790.

5. European Association for the Study of the Liver. EASL clinical practice guidelines: management of alcohol-related liver disease. $\mathrm{J}$ Hepatol 2018;69:154-181.

6. Maddrey WC, Boitnott JK, Bedine MS, Weber FL Jr, Mezey E, White RI Jr. Corticosteroid therapy of alcoholic hepatitis. Gastroenterology 1978;75:193-199.

7. Kamath PS, Wiesner RH, Malinchoc M, et al. A model to predict survival in patients with end-stage liver disease. Hepatology 2001;33:464-470.

8. Dominguez M, Rincón D, Abraldes JG, et al. A new scoring system for prognostic stratification of patients with alcoholic hepatitis. Am J Gastroenterol 2008;103:2747-2756.

9. Forrest EH, Evans CD, Stewart S, et al. Analysis of factors predictive of mortality in alcoholic hepatitis and derivation and validation of the Glasgow alcoholic hepatitis score. Gut 2005;54:11741179.

10. Lee M, Kim W, Choi Y, et al. Spontaneous evolution in bilirubin levels predicts liver-related mortality in patients with alcoholic hepatitis. PLoS One 2014;9:e100870.

11. Louvet A, Naveau S, Abdelnour M, et al. The Lille model: a new tool for therapeutic strategy in patients with severe alcoholic hepatitis treated with steroids. Hepatology 2007;45:1348-1354.

12. Mathurin P, Duchatelle V, Ramond MJ, et al. Survival and prognostic factors in patients with severe alcoholic hepatitis treated with prednisolone. Gastroenterology 1996;110:1847-1853.

13. Chedid A, Mendenhall CL, Tosch T, et al. Significance of megamitochondria in alcoholic liver disease. Gastroenterology 1986;90:1858-1864.

14. Katoonizadeh A, Laleman W, Verslype C, et al. Early features of acute-on-chronic alcoholic liver failure: a prospective cohort study. Gut 2010;59:1561-1569.

15. Spahr L, Rubbia-Brandt L, Genevay M, Hadengue A, Giostra E. Early liver biopsy, intraparenchymal cholestasis, and prognosis in patients with alcoholic steatohepatitis. BMC Gastroenterol 2011;11:115.

16. Mookerjee RP, Lackner C, Stauber R, et al. The role of liver biopsy in the diagnosis and prognosis of patients with acute deterioration of alcoholic cirrhosis. J Hepatol 2011;55:1103-1111.

17. Altamirano J, Miquel R, Katoonizadeh A, et al. A histologic scor- ing system for prognosis of patients with alcoholic hepatitis. Gastroenterology 2014;146:1231-1239.

18. Garcia-Tsao G, Sanyal AJ, Grace ND, Carey W; Practice Guidelines Committee of the American Association for the Study of Liver Diseases; Practice Parameters Committee of the American College of Gastroenterology. Prevention and management of gastroesophageal varices and variceal hemorrhage in cirrhosis. Hepatology 2007;46:922-938.

19. Moore KP, Wong F, Gines P, et al. The management of ascites in cirrhosis: report on the consensus conference of the International Ascites Club. Hepatology 2003;38:258-266.

20. Runyon BA; Practice Guidelines Committee, American Association for the Study of Liver Diseases (AASLD). Management of adult patients with ascites due to cirrhosis. Hepatology 2004;39:841-856.

21. Ferenci P, Lockwood A, Mullen K, Tarter R, Weissenborn K, Blei AT. Hepatic encephalopathy: definition, nomenclature, diagnosis, and quantification: final report of the working party at the 11th World Congresses of Gastroenterology, Vienna, 1998. Hepatology 2002;35:716-721.

22. Colmenero J, Bataller R, Sancho-Bru P, et al. Hepatic expression of candidate genes in patients with alcoholic hepatitis: correlation with disease severity. Gastroenterology 2007;132:687-697.

23. Kang L, Chen W, Petrick NA, Gallas BD. Comparing two correlated $\mathrm{C}$ indices with right-censored survival outcome: a one-shot nonparametric approach. Stat Med 2015;34:685-703.

24. Andrade P, Silva M, Rodrigues S, Lopes J, Lopes S, Macedo G. Alcoholic hepatitis histological score has high accuracy to predict 90-day mortality and response to steroids. Dig Liver Dis 2016;48:656-660.

25. Rincon D, Lo Iacono 0, Ripoll C, et al. Prognostic value of hepatic venous pressure gradient for in-hospital mortality of patients with severe acute alcoholic hepatitis. Aliment Pharmacol Ther 2007;25:841-848.

26. Dunn W, Jamil LH, Brown LS, et al. MELD accurately predicts mortality in patients with alcoholic hepatitis. Hepatology 2005;41:353-358.

27. Lu XL, Luo JY, Tao M, et al. Risk factors for alcoholic liver disease in China. World J Gastroenterol 2004;10:2423-2426.

28. Eng MY, Luczak SE, Wall TL. ALDH2, ADH1B, and ADH1C genotypes in Asians: a literature review. Alcohol Res Health 2007;30:22-27.

29. Horvath B, Allende D, Xie H, et al. Interobserver variability in scoring liver biopsies with a diagnosis of alcoholic hepatitis. Alcohol Clin Exp Res 2017;41:1568-1573. 